\title{
IDOSOS EM INSTITUIÇÃO DE LONGA PERMANÊNCIA E RELAÇÕES FAMILIARES
}

\author{
ELDERLY IN LONG TERM INTITUTION AND FAMILY RELATIONS
}

\section{Fernanda Monteiro Cataneo ${ }^{\mathrm{a}^{*}}$, Tuane Maiara Cardozo ${ }^{\mathrm{b}^{*}}$, Arlene Laurenti Monterrosa Ayala ${ }^{\mathrm{c}^{*}}$}

\author{
fernanda_cataneo@outlook.com ${ }^{\mathrm{a}}$, tucarover@hotmail.com ${ }^{\mathrm{b}}$, alayala@bol.com.br ${ }^{\mathrm{c}}$ \\ Instituto Superior e Centro Educacional Luterano Bom Jesus/Ielusc, Joinville (SC)*, Universidade Federal de \\ Santa Catarina* \\ Data de submissão: 20/01/2019 \\ Data de aceite: 18/02/2019
}

\section{RESUMO}

Introdução: As condições de vida do idoso em instituições de longa permanência tem sido objeto de pesquisas visto à falta de suporte, sobretudo de natureza afetiva e de apoio familiar. Objetivo: Este estudo teve por objetivo conhecer as características socioeconômicas e de proximidade familiar dos idosos que vivem em uma instituição de longa permanência localizada em Joinville, Santa Catarina. Materiais e Métodos: Trata-se de um estudo descritivo de caráter exploratório que foi realizado com dez idosos. Os dados foram coletados por meio de um questionário semiestruturado. As variáveis investigadas consideraram as características socioeconômicas, aspectos relacionados à saúde, à família e a instituição de longa permanência. Resultados: A idade dos idosos variou entre 66 a mais de 80 anos. A maioria era do sexo masculino possuía ensino médio completo e referiu-se a problemas de saúde. O tempo de institucionalização dos idosos variou de 4 meses a 10 anos e motivo mais frequente para a institucionalização foi a dependência física. Mais da metade dos entrevistados tiveram resposta positivas sobre gostar de estar na Instituição de Longa Permanência. Conclusões: A institucionalização do idoso pode ser geradora de sofrimento, mas também pode constituir-se um ambiente de conformação de vínculos afetivos. O idoso atribui considerável importância aos cuidados, o que impõe aos profissionais uma atenção que abranja os vários aspectos da vida da pessoa envelhecida. Em razão do envelhecimento populacional, seria desejável que fossem realizados estudos que aprofundassem a analise de outros fatores que poderiam estar contribuindo para qualidade de vida dos idosos institucionalizados.

Palavras-chave: Idoso, Instituição de Longa Permanência para Idosos, relações familiares.

\section{ABSTRACT}

Introduction: The living conditions of the elderly in long-term institutions have been the object of research, given the lack of support, especially affective and family support. Objective: This study aims to know the socioeconomic characteristics and the family nearness of the elderly that live in a long-term institution located in Joinville, Santa Catarina. Materials and Methods: This is an exploratory descriptive study that will be performed with ten elderly people. Data were collected through a semi-structured questionnaire. The studied variables considered the socioeconomic characteristics, aspects related to health, family and the institution of long stay. Results: The age of the elderly ranged from 66 to over 80 years. Most were male, had completed high school and referred to health problems. The time of institutionalization of the elderly ranged from 4 months to 10 years, and the most frequent reason for institutionalization was physical dependence. More than half of the interviewees had a positive answer about being in the Long Stay Institution. Conclusions: The institutionalization of the elderly can be a source of suffering, but it can also be an environment for the formation of affective bonds. The elderly attach considerable importance to care, which imposes on care professionals that cover the several aspects of the aged person's life. Due to the aging of the population, it would be desirable to carry out studies that deepen the analysis of other factors that could be contributing to the quality of life of the institutionalized elderly.

Keywords: Family relations, aged, Homes for the Aged. 


\section{Introdução}

O envelhecimento faz parte da realidade da maioria das sociedades. O mundo está envelhecendo. Tanto isso é verdade que se estima para o ano de 2050 que existam cerca de dois bilhões de pessoas com sessenta anos e mais no mundo ${ }^{1}$.

A alteração na estrutura etária da população ocorre em razão da redução das taxas de mortalidade, fecundidade e o aumento da esperança de vida, o que da origem ao envelhecimento populaciona ${ }^{2}$.

No Brasil, estima-se que até 2060 , a população idosa represente 70,5 milhões de pessoas. Em termos absolutos, as novas projeções de população, indicam que o número de jovens poderia diminuir rapidamente, de pouco mais de 52 milhões, em 2010, para 28 milhões, em 2060; enquanto que a população idosa poderia aumentar durante todo o período, passando de 14 milhões, em 2010, para 70,5 milhões, em $2060^{2}$.

A Organização Pan-Americana de Saúde (OPAS) define envelhecimento como um processo individual, irreversível, não patológico, de deterioração de um organismo maduro, de maneira que o tempo o torne menos capaz de enfrentar ao estresse do ambiente, tornando-o mais suscetível a problemas de saúde e até a morte ${ }^{3}$.

O maior desafio na atenção à pessoa idosa é conseguir contribuir para que elas possam viver sua vida com a máxima qualidade. Dessa forma é necessário que a família do idoso, preocupe-se com o cuidado com este indivíduo.

A lei $\mathrm{n}^{\circ} 10741$ de outubro de 2003, que dispõe sobre o Estatuto do Idoso em seu artigo $3^{\circ}$ cita que a família juntamente com a sociedade e poder público devem assegurar a pessoa idosa o direito à vida, $\mathrm{o}$ atendimento a suas necessidades básicas, a liberdade, a cidadania, a dignidade e a convivência familiar e comunitária ${ }^{4}$.

Nos últimos anos, as instituições de longa permanência para idosos (ILPIs), popularmente conhecidas como asilos, têm sido a alternativa adotada pelas famílias que, por razões diversas, não podem, não conseguem, ou não desejam cuidar de seu familiar idoso ${ }^{5}$.

Embora a família ainda seja uma alternativa de suporte ao idoso, autores referem que existem inúmeras razões que interferem na permanência dos idosos junto aos seus familiares, dentre eles, o conflito familiar, o aparecimento de agravos que geram a dependência do idoso, membros das famílias ocupados em suas obrigações cotidianas, a oferta de cuidados e companhia, além de um espaço de convivência e socialização ${ }^{6-8}$.

Apesar dos idosos referirem que necessitam de mais atenção, afeto e que a família é importante no contexto de vida de cada um deles, estudos têm demonstrado que o asilamento leva ao distanciamento progressivo entre os idosos e seus familiares, sendo que em determinados situações ocorre o afastamento completo da família ${ }^{8,9}$.

O estudo de Azeredo e Afonso ${ }^{10}$, realizado com idosos que vivem em ILPIs e em seus próprios domicílios demonstrou que os idosos que vivem com suas famílias, são aqueles que sentem menos solidão. Os que sentem muitas vezes ou quase sempre solidão são os idosos institucionalizados, os viúvos e divorciados. Do mesmo modo, o estudo de Rabelo e Neri ${ }^{11}$ confirma que a família se associa, em grande parte, a melhor qualidade de vida dos idosos. Cita a importância das interações familiares, a organização e o apoio familiar.

Assim, é possível dizer que os laços familiares dos idosos institucionalizados é um problema com o qual os profissionais de enfermagem irão lidar no dia-a-dia profissional, uma vez que podem desempenhar funções numa instituição de longa permanência.

Durante os estágios acadêmicos as autoras tiveram a oportunidade de desenvolver atividades de cuidado ao idoso em uma instituição de longa permanência, onde foi possível observar as dificuldades na manutenção da proximidade do idoso a sua família e o desejo por parte do idoso de não estar ali. Constatou-se que alguns idosos gostariam de ter seus familiares próximos, outros idosos, no entanto, não mostraram tal desejo e referiram sentir-se bem na ILPI. A percepção diferente entre os idosos em relação a estes dois aspectos motivaram-nos a abordar esta problemática.

Desta forma, este estudo, pretende por meio de um questionário semiestruturado conhecer as características socioeconômicas e de proximidade familiar dos idosos que vivem em uma instituição de longa permanência localizada 
em Joinville, Santa Catarina. Tais informações poderão facilitar a implementação dos cuidados na assistência a essa população.

\section{Metodologia}

Foi desenvolvido um estudo descritivo, exploratório com a participação de 10 idosos de uma Instituição de Longa Permanência (ILPI), na cidade de Joinville/SC.

A ILPI tem caráter privado, atende 21 idosos. $\mathrm{Na}$ instituição são admitidos idosos maiores de 60 anos, com alteração cognitiva controlada, independentes ou que apresentem no máximo dependência para até três autocuidados para a vida diária, como: dificuldades para alimentarse, problemas na mobilidade e dificuldades para realizar a higiene corporal.

Oestudo foi norteado pela Resolução 466/12 do Conselho Nacional de Saúde $(\mathrm{CNS})^{12}$, que trata das Diretrizes e Normas Regulamentadoras de Pesquisas envolvendo seres humanos e foi submetido à apreciação do comitê de ética, sendo aprovado sob o protocolo no 2.611.796.

Foram convidados a participar do estudo, todos os idosos, residentes no local, com idade igual ou superior a 60 anos, lúcidos, orientados, sem dificuldade de comunicação e que concordaram em participar da investigação mediante a assinatura do Termo de Consentimento Livre e Esclarecido (TCLE). Tais critérios foram adotados em razão da necessidade de perceber a importância dada pelo idoso à presença da família após a institucionalização. Foram excluídos 10 indivíduos por apresentarem problemas cognitivos importantes e 1 (hum) por ter manifestado o desejo de não participar do estudo.

O convite ocorreu de maneira individualizada, e na ocasião foram explicados os objetivos da pesquisa, seus critérios científicos e a garantia do anonimato. A coleta de dados aconteceu nos dias agendados com os entrevistados entre os meses de junho e julho de 2018.

Utilizou-se como técnica de coleta de dados um questionário com perguntas abertas e fechadas especialmente desenvolvido para o estudo. Com o objetivo de verificar a forma adequada de formulação das perguntas, foram aplicados três pré-testes com idosos residentes em outra ILPI. As perguntas tiveram por objetivo conhecer as características socioeconômicas, os aspectos relacionados à saúde e à família dos entrevistados e a ILPI. Quanto às características socioeconômicas - Qual é a sua idade? (60 a 65 anos; 66 a 70 anos; 71 a 75 anos; 76 a 80 anos; mais de 80 anos), Sexo (masculino; feminino), Escolaridade (ensino fundamental completo; ensino fundamental incompleto; ensino médio completo; ensino médio incompleto; nível superior). Em relação aos aspectos relacionados à saúde - Tem algum problema de saúde? (sim; não); Qual(is)?, Como sente sua saúde? (excelente; boa; regular; ruim). Sobre a família - Quanto tempo esta na ILPI?, Veio por livre espontânea vontade? (sim; não), Se não, quem lhe trouxe?. Em relação a família - Tem filhos? (sim; não), Se sim, quantos?, Sua família vem lhe visitar? (sim; não), Quem vem lhe visitar?, Com que frequência? Tem apoio da família? (sim; não), Tem amigos? (sim; não),Se sim, eles lhe visitam? (sim; não), Gosta das visitas? (sim; não), Sua família, amigos ou outros passeiam com o senhor(a)? (sim; não), Quando tem alguma necessidade quem o senhor(a) chama?, O senhor (a) gosta de estar aqui?.

A partir da pergunta aberta norteadora: o que o senhor (a) acha de estar aqui na ILPI ?, buscouse compreender a percepção e os sentimentos do idoso sobre sua permanência na ILPI. O registro das respostas foi transcrito no decorrer da entrevista. Este procedimento permitiu a reprodução das respostas com uma maior precisão.

As respostas das perguntas fechadas foram organizadas em programa Word, desenhado para o estudo. As variáveis pertinentes às características sociodemográficas, as concernentes à saúde e algumas questões relacionadas à família foram apresentadas a partir da análise descritiva dos dados por meio do cálculo das frequências absolutas (n) e relativas (\%).

Em relação às respostas da pergunta aberta sobre o que o idoso achava de estar na ILPI, consideraram-se como respostas positivas os sentimentos de apoio familiar, aceitação, segurança, de sentir-se cuidado, e de sentir-se bem. Como negativos, os sentimentos de solidão, abandono, revolta, conflito familiar, insegurança, 
incapacidade física e o desejo de não estar na ILPI. Para a análise dos achados comparouse os resultados encontrados e as informações presentes na literatura científica. Tal comparação teve por objetivo identificar discordâncias ou similaridades com os achados, apesar das diferentes circunstâncias.

\section{Resultados}

A idade dos idosos variou entre 66 a mais de 80 anos; $20 \%$ encontravam-se na faixa etária entre 66 a 70 anos; $30 \%$ entre 71 a 75 anos; e $50 \%$ com idades acima de 80 anos. A maioria (80\%) dos participantes era do sexo masculino.

Em relação ao grau de escolaridade dos idosos estudados, foram encontrados os percentuais de $50 \%$ para o ensino médio completo, $30 \%$ para o ensino fundamental completo e com nível superior e analfabeto $10 \%$ cada.

No que tange aos aspectos relacionados à saúde, $90 \%$ referiu possuir problemas de saúde, entretanto, a maioria (60\%) declarou sentir-se fisicamente bem. A prevalência de hipertensão arterial esteve presente entre $50 \%$ e a diabetes mellitus em 30\% dos idosos entrevistados.

O tempo de institucionalização dos idosos variou de 4 meses a 10 anos, sendo que $70 \%$ residiam na instituição entre 1 a 3 anos. $\mathrm{O}$ motivo mais frequente para a institucionalização foi a dependência física (40\%), e os filhos (60\%) foram os responsáveis pelo asilamento. A metade dos idosos possui de 1 a 3 filhos. A maioria (80\%) das visitas é realizada pelos filhos, e a frequência com que elas ocorrem, em grande parte, é quinzenalmente. Todos referiram gostar de receber visita e quando perguntados o(s) motivo(s) pelo(s) qual(is) gostavam de recebê-las, prevaleceu a necessidade da conversa com alguém (50\%), outra razão expressa pelos entrevistados foi porque recebiam "coisinhas" (30\%). Por fim, os sentimentos de afeto e amor justificaram o desejo das visitas para metade dos idosos. Os filhos têm sido responsáveis, em grande medida, em atender as necessidades dos idosos quando demandados por eles. (Tabela 1).

Tabela 1 - Descrição das características relacionadas à família e ao processo de institucionalização dos idosos. Joinville/SC, 2018.

\begin{tabular}{lc}
\hline Variável & n (\%) \\
\hline Tempo na ILP & $1(10)$ \\
$<1$ ano & $6(70)$ \\
1 a 3 anos & $2(20)$ \\
4 a 6 anos & $1(10)$ \\
7 a 10 anos & \\
Veio por livre e espontânea vontade & $3(30)$ \\
Sim & $7(70)$ \\
Não & \\
Motivo da institucionalização & $2(20)$ \\
A família trouxe & $4(40)$ \\
Dependência física & $1(10)$ \\
Achou melhor & $1(10)$ \\
Porque os filhos trabalham & $1(10)$ \\
Porque os filhos não tinham paciência & $1(10)$ \\
Tranquilidade &
\end{tabular}




\section{Recebimento de visitas \\ Sim \\ $10(100)$ \\ Não \\ Quem visita \\ Filhos, neta, nora \\ $4(40)$ \\ Filho \\ Irmãos \\ A frequência das visitas \\ Diariamente \\ Semanalmente \\ Quinzenalmente \\ Quando podem \\ Gosta de receber as visitas \\ Sim \\ Não \\ Motivação de gostar das visitas \\ Conversar \\ Amor \\ Trazem coisinhas

Mais da metade dos entrevistados (80\%) relatou possuir amigos, destes, 50\% apontaram como amigos os idosos que residiam com eles na própria instituição. A maioria (60\%) referiu ter o habito de sair com frequência da ILP para passear e/ou almoçar com os filhos. Quanto aos demais entrevistados, $20 \%$ não saem da instituição e sugerem que os filhos não os levam para passear e os $20 \%$ restante alegam possuírem dificuldades na deambulação.

Foi constatado a partir das respostas à pergunta aberta, que seis dos dez entrevistados tiveram resposta positivas sobre gostar de estar na ILP, e a razão alegada de quatro deles foi o tratamento e os cuidados adequados destinados pelos profissionais da instituição. Os outros dois aludiram como razões principais à possibilidade de estabelecer amizades e conversas entre eles. Em relação aos quatro que tiveram manifestações negativas sobre gostar de estar na ILP, três citaram que gostariam de estar em suas próprias casas e apenas 1 (hum) referiu o desejo de estar próximo a familiares.

\section{Discussões}

As idades prevalentes entre os participantes foram acima de 80 anos. Este resultado pode ser atribuído pelo aumento da longevidade entre os brasileiros ${ }^{13}$. Houve o predomínio do sexo masculino entre os entrevistados. Este achado não é frequentemente descrito na literatura, pois na maioria dos estudos o percentual da população feminina nas ILPI é numericamente superior em relação aos homens ${ }^{14,15}$. Todavia, dois estudos realizados em ILPI em Pernambuco ${ }^{16} \mathrm{e}$ Distrito Federa $1^{17}$ encontraram maior percentual do sexo masculino entre os idosos investigados, respectivamente, $55,9 \%$ e $58,8 \%$.

Com relação à escolaridade prevaleceu o ensino médio completo entre os entrevistados. Entretanto, este resultado diverge de outros estudos que apontam para a baixa escolaridade dos idosos na ILPIs tanto nas instituições privadas como publicas, com predomínio para os que cursaram primário incompleto e analfabetos ${ }^{18,19}$. Dados do ultimo censo 2010 revelam que em Santa 
Catarina o percentual de pessoas sem instrução ou com o fundamental incompleto decresceu, houve uma elevação expressiva no nível de instrução no Estado e o percentual de pessoas com pelo menos o ensino médio completo aumentou aproximadamente $16 \%{ }^{20}$

A maioria dosidosos referiu ter pelo menos uma doença crônica (80\%), sendo que a hipertensão arterial prevaleceu. Outros estudos demonstram uma importante frequência de doenças crônicas entre os idosos institucionalizados. Alencar et al. ${ }^{14}$ encontraram $57,4 \%$ de idosos que referiram doenças crônicas. No estudo feito por Lima et al, ${ }^{15}$ observou-se que $70,6 \%$ possuíam doenças crônicas e destas $41,2 \%$ era hipertensão. Chama a atenção que em relação à autopercepção de saúde, neste estudo a maioria dos idosos institucionalizados considerou sua saúde como boa. Este resultado é corroborado pelos dados encontrados no estudo de Alencar et al. ${ }^{14}$ que verificou $61,7 \%$ dos idosos com autopercepção boa sobre suas condições de saúde. Atualmente, a autopercepção da saúde, é um dos indicadores mais utilizados em pesquisas gerontológicas. Seu uso é justificado porque a pior percepção de saúde é um preditor consistente de declínio funcional ou morte do idoso ${ }^{21}$.

Com relação à permanência na instituição a maioria dos idosos residia entre 1 a 3 anos, sendo que este achado se aproximou do estudo de Azevedo et al. ${ }^{23}$ realizado em uma ILPI, na cidade de Natal-RN que apontou para um tempo de no máximo 4 anos de institucionalização. Entretanto, diverge de outros estudos que apontam para períodos maiores de residência dos idosos nas instituições ${ }^{5,18,22}$. Observou-se que a institucionalização compulsória foi a que prevaleceu nesta investigação, sendo que as pesquisas demonstram que a institucionalização ocorre em razão de vários motivos, entre os quais, dependência física como observado neste estudo, além de determinantes sociais e familiares ${ }^{5,14}$, 24, 25. Foi constatado na pesquisa que a decisão de institucionalização, ficou a cargo dos filhos dos idosos. As observações de vários estudos coincidem com este achado indicando a realidade de precarização dos vínculos, muito em razão de dificuldades dos membros da família para dar ao idoso um suporte adequado ${ }^{25,26}$. Sugerindo, portanto, a ausência de infraestrutura adequada para responder às demandas dessa população em relação a atendimento domiciliário e ambulatorial dos serviços de saúde.

Foi observado que os participantes por unanimidade referiram gostar de receber visitas de amigos e familiares. E a necessidade de conversar foi referida pela metade dos entrevistados. Brandão e Zatt ${ }^{27}$ atestam que os relacionamentos sociais são necessários na vida das pessoas em qualquer fase da vida e contribuem para dar sentido a ela. Para os autores, embora, na velhice já se tenha aprendido muitas habilidades para bem viver, o contato com outras pessoas é imprescindível nesta fase da vida. Da mesma forma Figueiredo et al. ${ }^{28}$ atestam que as relações de amizade atenuam as situações de risco para tentativas de suicídio entre os idosos, por isso o cultivo de amizades e os vínculos familiares é visto como um fator que protege de maneira importante contra a ideação e tentativas de suicídio. Chama a atenção o fato do vinculo de amizade entre os próprios idosos na instituição em estudo. $\mathrm{O}$ que foi constatado é que os idosos que compartilham o convívio experimentam o estreitamento de seus vínculos afetivos. De acordo com Evangelista et al. ${ }^{29}$ o dia-adia com os semelhantes que possuem experiências da mesma época convívio de idosos faz com que os idosos enxerguem a ILP como um lugar agradável e preferem viver ali a viverem sozinhos.

A qualidade de vida do idoso pode ser avaliada, em parte, pela satisfação que tem ao estar naquele ambiente. Neste estudo a maioria dos idosos enxerga a institucionalização de forma positiva em razão dos cuidados recebidos pelos profissionais da instituição. Figueiredo et $\mathrm{al}^{28}$ citam que cuidados que melhorem a qualidade de vida dos idosos contribuem para que eles pensem em novos projetos de vida, o que é fundamental para mantê-los com boa autoestima. Porem, 40\% dos idosos referiu não gostar de estar na ILP e que gostariam de estar em suas próprias casas ou com seus familiares. Para Silva et al..$^{30}$ as manifestações negativas dos idosos quanto a permanência em ILP estão associadas ao sentimento de solidão e ao abandono. Segundo os autores, o idoso ao manifestar estes tipos de desejos indica que a rede de relações existentes não é satisfatória. Neste ponto é necessário chamar atenção para alguns estudos que apontam como fatores de risco 
para suicídio entre os idosos, entre eles, os laços familiares distantes ou conflituosos, o abandono físico e afetivo e as dificuldades de adaptação à vida institucional ${ }^{28,31}$.

\section{Considerações Finais}

Os achados deste estudo encontram-se em consonância com os dados da literatura, que apontam para os sentimentos conflitantes do idoso acerca do seu cotidiano em instituições de longa permanência. Se por um lado, a institucionalização do idoso pode ser geradora de sofrimento e de abandono, por outro, pode constituir-se um ambiente de conformação de novos vínculos afetivos;

É necessário ressaltar a importância atribuída pelos idosos ao cuidado, impondo aos profissionais uma atenção única e individual e que abranja os vários aspectos da vida da pessoa envelhecida, como a sua saúde e suas interações sociais;

Por fim, por se tratar de um problema de grande magnitude, em razão do envelhecimento populacional, seria desejável que fossem realizados estudos que aprofundassem a analise com maior precisão de outros fatores que poderiam estar contribuindo para qualidade de vida dos idosos em ILP.

\section{Referências}

1. Melo JV, Santos ALM, Faria LR, Alves WA, Rezende AAA, Calábria L, Karen C. Perfil de saúde dos idosos atendidos nas unidades básicas de saúde da família (UBSF) em Ituiutaba, Minas Gerais. Rev. Aten. Saúde, 2017; 53: 66-75.

2. Flores, LPO. O envelhecimento da população brasileira. Redeca, 2015; 1:86-100.

3. World Health Organization. Organização Pan-Americana da Saúde. Envelhecimento ativo: uma política de saúde. Brasília; 2005.

4. Brasil. Casa Civil. Lei no 10741 de outubro de 2003. Dispõe sobre o Estatuto do Idoso e dá outras providências. Brasília, 2003. [citado mar 2018]. Disponível em: <http:// www.planalto.gov.br/ccivil_03/leis/2003/110.741.htm>.
5. Martins EF, Guimarães, FP. Perfil dos idosos de uma instituição delonga permanência de uma cidade dointerior de Minas Gerais. Rev. Bras. Ciências da Vida [Internet] Ago 2017 [citado mar 2018]; 5(2). ISSN 2525-359X. Disponível em: http://jornal.faculdadecienciasdavida. com.br/index.php/RBCV/article/view/538.

6. Martins, E. Constituição e significação de família para idosos institucionalizados: uma visão histórico-cultural do envelhecimento. Estudos e Pesquisas em Psicologia [Internet]. 2013 [citado mar 2018]; 13(1): 215-236. Disponível em: http://www.redalyc.org/articulo. oa? id=451844510014.

7. Bentes ACO, Pedroso JS, Maciel CAB. O idoso nas instituições de longa permanência: uma revisão bibliográfica. Aletheia [Internet]. Dez 2012 [citado set 2018]; (38-39): 196-205. Disponível em: http://pepsic. bvsalud.org/scielo.php?script=sci_arttext\&pid=S141303942012000200016\&lng=pt.

8. Rissardo LK; Furlan MCR; Grandizolli G; Marcon SS; Carreira L. Concepção e sentimentos de idosos institucionalizados sobre família. Ciência, Cuidado e Saúde [Internet] 2011 [citado mar 2018];10(4): 682-689, 2011. Disponível em: http://www.periodicos.uem.br/ojs/ index.php/CiencCuidSaude/article/view/18311/pdf.

9. Santos NO; Beuter M; Girardon-Perlini NMO; Paskulin LMG; Leite MT; Budó MLD. Percepção de trabalhadores de uma instituição de longa permanência para idosos acerca da família. Texto Contexto Enferm [Internet]. 2014 [citado mar 2018]; 23(4): 971-978. Disponível em: http:// www.scielo.br/pdf/tce/v23n4/pt_0104HYPERLINK "http://www.scielo.br/pdf/tce/v23n4/pt_0104-0707tce-23-04-00971.pdf”0707-tce-23-04-00971.pdf.

10. Azeredo ZAS; Afonso MAN. Solidão na perspectiva do idoso. Rev. bras. geriatr. geronto. [Internet]. 2016 Abr [citado set 2018]; 19(2): 313-324. Disponível em: http:// www.scielo.br/scielo.php?script=sci_arttext\&pid=S180998232016000200313\&lng=en. http://dx.doi. org/10.1590/1809-98232016019.150085

11. Rabelo DF, Neri AL. Arranjos domiciliares, condições de saúde física e psicológica dos idosos e sua satisfação com as relações familiares. Rev. bras. geriatr. geronto. [Internet]. 2015 Set [citado set 2018]; 18(3): 507-519. Disponível em: $\quad$ http://www.scielo.br/scielo.php?script=sci_ arttext\&pid $=$ S1809-98232015000300507\&lng=en. http://dx.doi.org/10.1590/1809-9823.2015.14120.

12. Brasil. Conselho Nacional de Saúde. Resolução $\mathrm{n}^{\circ}$ 466, de 12 de dezembro de 2012.Diretrizes e normas regulamentadoras de pesquisas envolvendo seres humanos. Diário Oficial da União [da] República Federativa do Brasil, 150(112), Seção 1, 2013. [Citado fev 2018] Disponível em: http://sintse.tse.jus.br/ documentos/2013/Jun/13/cns-resolucao-no-466-de-12dedezembro-de-2012. 
13. IBGE/Diretoria de Pesquisas. Coordenação de População e Indicadores Sociais. Gerência de Estudos e Análises da Dinâmica demográfica. Projeção da população do Brasil e Unidades da Federação por sexo e idade para o período 2010-2060.

14. Alencar MA, Bruck NNS, Pereira BC, Câmara TMM, Almeida RDS. Perfil dos idosos residentes em uma instituição de longa permanência. Rev. bras. geriatr. gerontol. [Internet] $2012 \mathrm{Dez}$ [citado set 2018]; 15(4): 785-796. Disponível em: http://www. scielo.br/scielo.php?script=sci_arttext\&pid=S180998232012000400017\&lng=en.

15. Souza ACC, Martins KA. Mudança do perfil de idosos de uma instituição de longa permanência nos últimos dez anos. Geriatr Gerontol Aging. [Internet] 2016 [citado set 2018]; 10(1):16-22. Disponível em: http://www.ggaging. com/details/68/pt-BR/change-in-the-profile-of-elderlyfrom-a-prolonged-stay-institution-in-the-last-ten-years.

16. Lima RMF, Amaral AKFJ, Aroucha EBL, Vasconcelos TMJ, Silva HJ, Cunha DA. Adaptações na mastigação, deglutição e fonoarticulação em idosos de instituição de longa permanência. Rev CEFAC. [Internet] 2009 [citado set 2018]; 11(3): 405-422. Disponível em: http://www. scielo.br/pdf/rcefac/v11s3/a17v11s3.pdf.

17. Castellar JI, Karnikowski MGO, Viana LG, Nóbrega OT. Estudo da Farmacoterapia Prescrita a Idosos em Instituição de Longa Permanência. Acta Med Port. 2007; 20: 97-105.

18. Rosa P, Glock L, Berlezi E, Rossato D, Rosa L. Perfil dos idosos residentes em instituições de longa permanência da região sul do país. RBCEH [Internet]. Ago 2012 [citado set 2018]; 8(1): 38-47. Disponível em: http://www. seer.upf.br/index.php/rbceh/article/view/870

19. Ferreira LL, Cochito TC, Caires F, Marcondes LP, Saad PC. Perfil sociodemográfico e funcional de idosos institucionalizados. Estud. Interdiscipl. Envelhec [Internet] 2012 [citado ago 2018]; 17(2):373-386 Disponível em: https://seer.ufrgs.br/RevEnvelhecer/ article/view/27641

20. Ministério do Planejamento, Orçamento e Gestão Instituto Brasileiro de Geografia e Estatística - IBGE Censo Demográfico 2010 Resultados gerais da amostra. 2012.

21. Santiago LM, Luz LL, Mattos IE, Gobbens RJJ. Adaptação transcultural do instrumento Tilburg Frailty Indicator (TFI) para a população brasileira. Cad. Saúde Pública [Internet]. Set 2012 [citado set 2018]; 28(9): 1795-1801. Disponível em: http://www. scielo.br/scielo.php?script $=$ sci_arttext $\&$ pid $=S 0102$ 311X2012000900018\&lng=en.
22. Cordeiro LM, Paulino JL, Bessa MEP, Borges CL, Leite SFP. Qualidade de vida do idoso fragilizado e institucionalizado. Acta paul. enferm. [Internet]. Ago 2015 [citado set 2018]; 28(4): 361-366. Disponível em: $\quad$ http://www.scielo.br/scielo.php?script=sci arttext\&pid $=$ S0103-21002015000400012\&lng=en. http://dx.doi.org/10.1590/1982-0194201500061.

23. Azevedo LM, Lima HHG, Oliveira KSA, Medeiros KFM, Gonçalves RG, Nunes VM, Piuvesam G. Perfil sociodemográfico e condições de saúde de idosos institucionalizados. Rev. Bras. Pesq. Saúde. [Internet] JulSet 2017 [citado set 2018]; 19(3): 16-23. Disponível em: http://periodicos.ufes.br/RBPS/article/view/19560.

24. Pinheiro NCG, Holanda VCD, Melo LA, Medeiros $\mathrm{AKB}$, Lima KC. Desigualdade no perfil dos idosos institucionalizados na cidade de Natal, Brasil. Ciênc. saúde coletiva [Internet]. Nov. 2016 [citado set 2018]; 21(11): 3399-3405. Disponível em: http://www. scielo.br/scielo.php?script=sci_arttext \&pid=S141381232016001103399\&lng=en. / http://dx.doi. org/10.1590/1413-812320152111.19472015.

25. Ferretti F, Soccol BF, Albrecht DC, Ferraz L. Viver a velhice em ambiente institucionalizado. Estud. interdiscipl. envelhec. [Internet] 2014 [citado set 2018]; 19(2): 423-437. Disponível em: https://seer.ufrgs.br/ RevEnvelhecer/article/view/42378.

26. Batista AS, Araújo AB. Intimidade e mercado: o cuidado de idosos em instituições de longa permanência. 2011 Sociedade e Estado, 26(1): 175-195.

27. Brandão VC, Zatt GC. Percepção de idosos, moradores de uma instituição de longa permanência de um município do interior do Rio Grande do Sul, sobre qualidade de vida. Aletheia [Internet] Jan-Abr 2015 [citado set 2018]; 46:90102. Disponível em: http://pepsic.bvsalud.org/scielo. php?script=sci_arttext\&pid=\$1413-03942015000100008.

28. Figueiredo AEB, Silva RM, Vieira LJES, Mangas RMN, Sousa GS, Freitas JS et al. É possível superar ideações e tentativas de suicídio? Um estudo sobre idosos. Ciênc. saúde coletiva. [Internet] Jun 2015 [citado set 2018]; 20(6):1711-1719. Disponível em: http://www.scielo.br/ $\mathrm{pdf} / \mathrm{csc} / \mathrm{v} 20 \mathrm{n} 6 / 1413-8123-\mathrm{csc}-20-06-1711 . p d f$.

29. Evangelista RA, Bueno AA, Castro PA, Nascimento JN, Araújo NT, Aires GP. Percepções e vivências dos idosos residentes de uma instituição asilar. Rev. esc. enferm. USP [Internet]. Dez 2014 [citado set 2018]; 48(spe2): 81-86. Disponível em: http://www. scielo.br/scielo.php?script $=$ sci_arttext $\&$ pid $=S 0080$ 62342014000800081\&lng=en. / http://dx.doi. org/10.1590/S0080-623420140000800013.

30. Silva LM, Moreira MASP, Bezerra VP, Almeida SA, Brenna S, Silva AO. Representações sociais sobre solidão por idosos institucionalizados. J. res.: fundam. care. [Internet] Dez 2014 [citado set 2018] 6(supl.):1-9 Disponível em: http://www.redalyc.org/pdf/5057/505750772001.pdf. 
31. Minayo MCS, Figueiredo AEB, Mangas RMN. O comportamento suicida de idosos institucionalizados: histórias de vida. Physis [Internet] Dez 2017 [citado set 2018]; 27(4): 981-1002. Disponível em: http://www.scielo. br/pdf/physis/v27n4/0103-7331-physis-27-04-00981. pdf.

Como citar este artigo:

Caetano FM, Cardozo TM, Ayala ALM. Idosos em instituição de longa permanência e relações familiares. Rev. Aten. Saúde. 2019;17(60):28-36. 